\title{
Discrimination of exudative pleural effusions based on multiple biological
} parameters

\author{
Z.D. Daniil*, E. Zintzaras”, T. Kiropoulos*, A.I. Papaioannou*, A. Koutsokera*, \\ A. Kastanis" and K.I. Gourgoulianis*
}

ABSTRACT: Pleural effusion is a common complication of various diseases. Conventional methods are not always capable of establishing the cause of pleural effusion, so alternative tests are needed. The aim of this study was to explore means of discriminating between different pleural effusion groups, malignant, parapneumonic and tuberculous, based on the combined function of seven biological markers.

Adenosine deaminase (ADA), interferon- $\gamma, \mathrm{C}$-reactive protein (CRP), carcinoembryonic antigen, interleukin-6, tumour necrosis factor- $\alpha$ and vascular endothelial growth factor concentration levels were measured in pleural fluid from 45 patients with malignant, 15 with parapneumonic and 12 with tuberculous pleural effusion. Receiver operating characteristic curve analysis, multinomial logit modelling and canonical variate analysis were applied to discriminate the pleural effusion groups.

The three groups could be discriminated successfully using the measured markers. The most important parameters for discrimination were ADA and CRP concentration levels. An individual with an ADA concentration level of $>45 \mathrm{U} \cdot \mathrm{L}^{-1}$ and a CRP concentration of $<4 \mathrm{mg} \cdot \mathrm{dL}^{-1}$ was more likely to belong to the tuberculous pleural effusion group, whereas one with an ADA concentration level of $<40 \mathrm{U} \cdot \mathrm{L}^{-1}$ and a CRP concentration of $>6 \mathrm{mg} \cdot \mathrm{dL}^{-1}$ was more likely to belong to the parapneumonic pleural effusion group, and one with a CRP concentration of $<4 \mathrm{mg} \cdot \mathrm{dL}^{-1}$ to the malignant pleural effusion group.

The combination of adenosine deaminase and C-reactive protein levels might be sufficient for discriminating between the three different groups of exudative pleural effusion: malignant, tuberculous and parapneumonic.

KEYWORDS: Adenosine deaminase, biological markers, C-reactive protein, discrimination and classification, diagnostic rule, pleural effusion

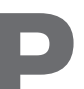
leural effusion can occur as a complication of many different diseases. It is a common clinical problem and it has been estimated that there are $>800,000$ cases $\cdot \mathrm{yr}^{-1}$ in the USA [1]. The diagnosis of pleural effusions remains a controversial issue in terms of cost to both patients and the healthcare system. Conventional methods are not always capable of establishing the cause of pleural effusion, and alternative tests permitting rapid and accurate diagnosis are greatly needed. Malignant disease involving the pleura and parapneumonic effusion are the leading causes of exudative pleural effusions. However, the diagnosis of tuberculous pleuritis should also be considered in any patient with an exudative pleural effusion.

A variety of biological markers have been proposed to facilitate differential diagnosis among the above-mentioned causes of pleural effusion, including pleural fluid concentrations of adenosine deaminase (ADA), interferon (IFN)- $\gamma$, a variety of tumour markers and cytokines, and C-reactive protein (CRP). Although there is a large body of literature on these biological markers and their utility in the diagnosis of pleural effusion, to date diagnosis is usually based on each individual marker separately.

The aim of the present study was to explore means of discriminating between different pleural effusion groups (malignant (MPE), parapneumonic (PPE) and tuberculous pleural effusion (TPE)), and to provide a classificationdiagnostic rule based on a function of seven markers (parameters): ADA, IFN- $\gamma, \mathrm{CRP}$, carcinoembryonic antigen (CEA), interleukin (IL)-6,

\section{AFFILIATIONS}

Depts of *Respiratory Medicine and ${ }^{\#}$ Biomathematics, University of Thessaly School of Medicine, Larissa, Greece.

CORRESPONDENCE

E. Zintzaras

Institute for Clinical Research and Health Policy Studies

Tufts-New England Medical Center Tufts University School of Medicine 750 Washington Street Tufts-NEMC \#63

Boston

MA 02111

USA

Fax: 16176368628

E-mail: zintza@med.uth.gr

Received:

September 272006

Accepted after revision: July 242007

STATEMENT OF INTEREST None declared. 
tumour necrosis factor (TNF)- $\alpha$ and vascular endothelial growth factor (VEGF).

\section{METHODS AND MATERIALS Subjects}

A total of 72 pleural fluid samples were collected prospectively from 72 patients at the Dept of Respiratory Medicine of the University of Thessaly Medical School (Larissa, Greece) between January and June 2005. The study group included 51 males and 21 females with a mean age of 65 yrs. Of these, 45 (62\%) had MPE, 15 (21\%) had PPE and 12 (17\%) had TPE. The study protocol was approved by the Ethics Committee of the University Hospital of Larissa (Larissa, Greece), and all subjects gave their written informed consent.

\section{Diagnostic criteria for pleural effusions}

The determination of the aetiology of the pleural effusions was based on the clinical presentation, appropriate diagnostic test results and response to treatment of each patient. Accordingly, effusions were classified into the following groups defined by predetermined criteria. 1) MPE, occurring secondary to lung cancer (diagnosed by the demonstration of malignant cells on cytological examination or in a biopsy specimen, or by histologically proven primary lung malignancy with the exclusion of any other cause of pleural effusion) and other malignancies (effusions that were clearly secondary to other malignancies, with exclusion of other causes for the development of pleural effusions; table 1). 2) TPE, diagnosed on the basis of the presence of either positive staining or culture for Mycobacterium tuberculosis in the pleural fluid, sputum or pleural biopsy specimen or typical caseating granulomas on pleural biopsy. 3) PPE, identified by the presence of pulmonary infections associated with acute febrile illness, pulmonary infiltrates, purulent sputum and response to antibiotic treatment; identification of the organism in the pleural fluid; or the presence of empyema, associated with the finding of frank pus in the pleural cavity.

\section{Sample collection and determination of ADA, CRP, IFN- $\gamma$, IL-6, TNF- $\alpha$, VEGF and CEA levels}

Samples were obtained from the first successful thoracentesis and before any treatment was initiated, immediately after each patient's admission. At the same time, $10 \mathrm{~mL}$ venous blood were obtained from each patient. Pleural fluid and blood samples were subsequently analysed for total cell count, differential cell count and levels of glucose, total protein and lactate dehydrogenase. All biochemical measurements were performed using standard commercially available methods (Olympus AU 600; Olympus Diagnostics, Lismeehan, Ireland),

\begin{tabular}{lc} 
TABLE 1 Aetiology of malignant pleural effusions \\
\hline Lung cancer & 31 \\
Ovary cancer & 1 \\
Renal cell carcinoma & 1 \\
Breast cancer & 7 \\
Prostate cancer & 2 \\
Gastric cancer & 3 \\
\hline & \\
\hline &
\end{tabular}

whereas cell counts were obtained by manual microscopy. Cytological examinations and both aerobic and anaerobic bacterial culture were performed on all pleural effusions. Aliquots of pleural fluid and blood samples were immediately centrifuged for $15 \mathrm{~min}$ at $1,500 \times g$ at $4{ }^{\circ} \mathrm{C}$ and the supernatants stored at $-80^{\circ} \mathrm{C}$ until further analysed by measurement of the above marker levels. ADA activity was measured by the colorimetric method of GIUSTI [2]. CRP measurements were performed by immunonephelometry with the Behring Nephelometer Analyzer II, using the N High Sensitivity kit (Dade Behring, Marburg, Germany). The appropriate control and standard sera were provided by the same company, and assays were performed according to the manufacturer's instructions. IL- 6 , VEGF and TNF- $\alpha$ concentration levels were measured using a commercially available ELISA kit (Biosource Europe, Nivelles, Belgium) according to the manufacturer's protocol. CEA concentration levels were determined using an electrochemiluminescence immunoassay on the Roche Modular E 170 analyser (Roche Diagnostics, Mannheim, Germany).

\section{Statistical methodology}

The discriminating quality of each biological marker was evaluated independently using receiver operating characteristic (ROC) curve analysis [3]. The ROC curve analysis was applied for two comparisons: PPE against TPE and MPE, and TPE against MPE and PPE. The quality of the biological markers was assessed based on the area under the curve (AUC). For each ROC curve, a cut-off point was determined as the value of the parameter that maximised the sum of specificity and sensitivity, weighting their significance equally.

Discrimination between the three study groups (MPE, TPE and PPE) based on all parameters simultaneously was investigated by fitting a multinomial logit model [4]. Multinomial logistic regression is useful for situations in which it is necessary to classify individuals based on values of a set of predictor variables (continuous and/or categorical). This type of regression is similar to logistic regression but is more general because the dependent variable is not restricted to two categories. In the present study, the aim was to classify individuals into the three pleural effusion groups based on the measured markers / parameters, which were continuous. The overall significance of parameters was tested using the likelihood ratio test; parameters with a p-value of $>0.05$ were omitted and then a parsimonious model was used. This procedure permits easier interpretation of the results without substantial loss of information. The parameters of the parsimonious model can be used for classifying individuals into their groups. The logit model estimates the probability $\left(p_{1}, p_{2}\right.$ and $\left.p_{3}\right)$ that an individual belongs to the MPE, TPE and PPE group, respectively. Each individual is classified in the group with the highest probability. The percentage of correctly classified individuals in each group was calculated, and the importance of the model was assessed based on misclassified individuals. The detection rate (DR; sensitivity), false-positive rate (FPR; 1specificity) and likelihood ratio (DR/FPR) of the significant parameters were also considered.

In order to provide a combination of parameter values for discriminating between the three groups, parameter values based on the cut-off points of the ROC curve analysis were entered into the logit model and the respective probabilities 
TABLE 2 Descriptive statistics of the seven parameters for each pleural effusion group

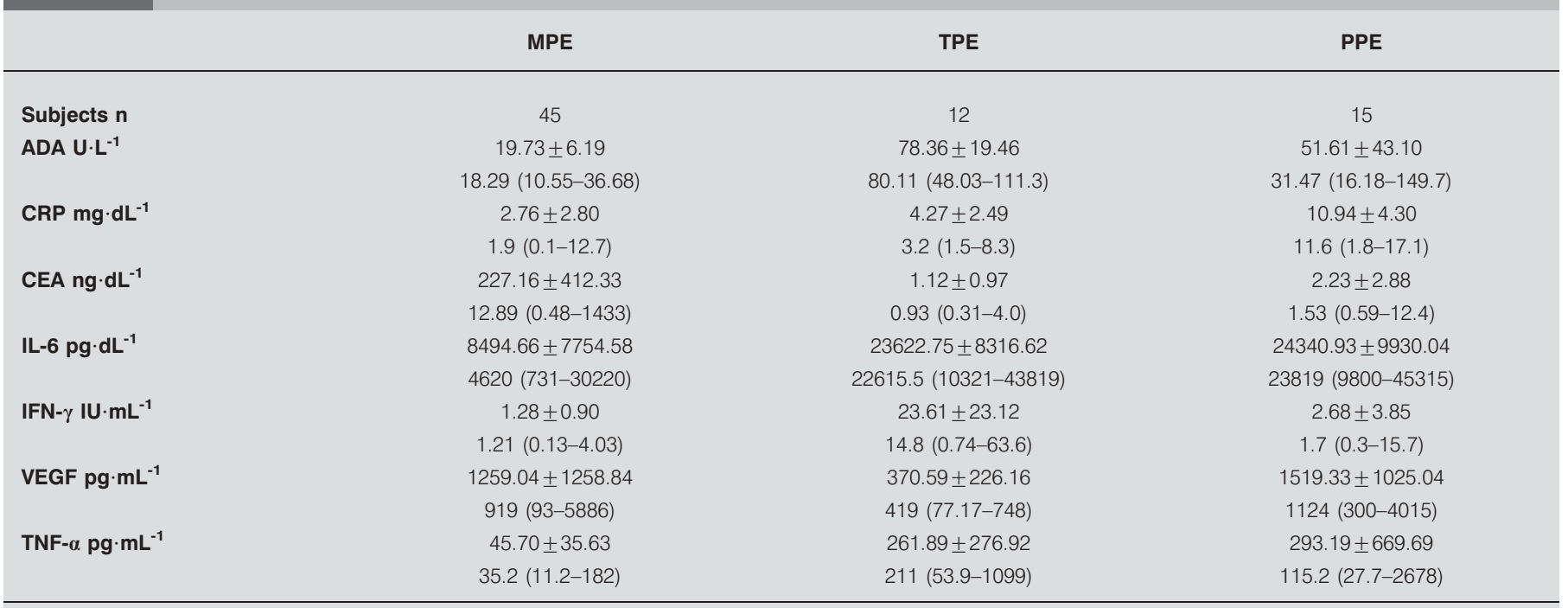

Data are presented as mean \pm SD and median (range). MPE: malignant pleural effusion; TPE: tuberculous pleural effusion; PPE: parapneumonic pleural effusion; ADA: adenosine deaminase; CRP: C-reactive protein; CEA: carcinoembryonic antigen; IL: interleukin; IFN: interferon; VEGF: vascular endothelial growth factor; TNF: tumour necrosis factor.

$\left(p_{i} ; i=1-3\right)$ were estimated. In addition, the three pleural effusion groups were discriminated using canonical variate (CV) analysis (CVA). CVA provides ordination of patients on the basis of their biological marker measurement (parameters). CVA examines the separations among a set of groups of units. For this purpose, CVA seeks the linear combinations of the $k$ parameters $(k=1-7)$, called CVs, that show the greatest between-group variation relative to their within-group variability. CV1 and CV2 are the eigenvectors of $W^{-0.5} B W^{-0.5}$, where $B$ is the between-group sums of squares and products (SSP) matrix, $W=\sum_{\mathrm{i}=1-18} W_{\mathrm{i}}$, and $W_{i}$ is the within-group $i$ SSP matrix. Two-dimensional ordination from CVA (CV1 versus CV2) usually accounts for most of the variation in the data $[5,6]$.

\section{RESULTS}

The descriptive statistics of the seven parameters for each pleural effusion group are shown in table 2. All parameters deviate from normality (except IL-6) but, after logarithmic transformation, most parameters appeared relatively normal, with the exception of CEA. The data were analysed raw since such results can be interpreted to clinicians more easily and the logit model for discriminant analysis may hold in the absence of normality [4], although any conclusions must be drawn cautiously. In addition, data transformation may mask the real effects of the parameters and their interactions [7]. Figure 1 shows typical distributions of ADA and CRP after logarithmic transformation. Table 3 shows the Spearman's correlation coefficients $(r)$ between the seven parameters. The nominal correlations between the parameters are generally low. However, the highest correlations were shown between TNF- $\alpha$ and ADA $(r=0.62)$, CEA $(r=-0.44)$ and IL-6 $(r=0.48)$, and between IL-6 and ADA ( $r=0.51)$ and CRP $(r=0.55)$.

The AUCs derived from the ROC curve analysis for each parameter are given in table 4 . In discriminating TPE from MPE and PPE, ADA gave the largest AUC (0.94), with a cut-off point of 42.2, and, in discriminating PPE from MPE and TPE, CRP provided the largest AUC (0.92), with a cut-off point of 5.5.

Only the parameters ADA $(p=0.01)$ and CRP $(p=0.05)$ were of significant importance to the simultaneous system after fitting the logit model. The fitting of the model, although not optimal, may be considered satisfactory, as can be seen from the halfnormal plot of the residuals (i.e. the difference between the observed and predicted probabilities; fig. 2). Thus, a parsimonious model was fitted including only the parameters ADA $(\mathrm{p}<0.01)$ and CRP $(\mathrm{p}<0.01)$. Table 5 shows individuals classified based on the logit model: four individuals with MPE were wrongly classified as having PPE, one individual with TPE was wrongly classified as having PPE, and three individuals with PPE were wrongly classified as having MPE (two cases) or TPE. The overall proportion of misclassified individuals was $11 \%$. The effect of adding additional markers to the significant parameters ADA and CRP was explored in a logit model (table 6). Combinations of up to four parameters were considered in this model since the aim was to investigate the utility of the minimum possible number of parameters; in addition, false-positive results were kept to minimum. Obviously, the more information included in the logit model, the lower the expected misclassification rate; however, the best trade-off between the number of parameter and the misclassification rate seems to be the model including the parameters ADA and CRP, although inclusion of IFN- $\gamma$ could be an alternative option. Table 7 shows the DR, FPR and likelihood ratio (DR/FPR) in consideration of ADA and CRP together and in combination with other parameters in the logit model. For ADA and CRP in this model, the likelihood ratio is greatest for TPE (likelihood ratio=54), suggesting that the two parameters may be much better at classifying TPE correctly, while minimising incorrect classification of the other two groups. 

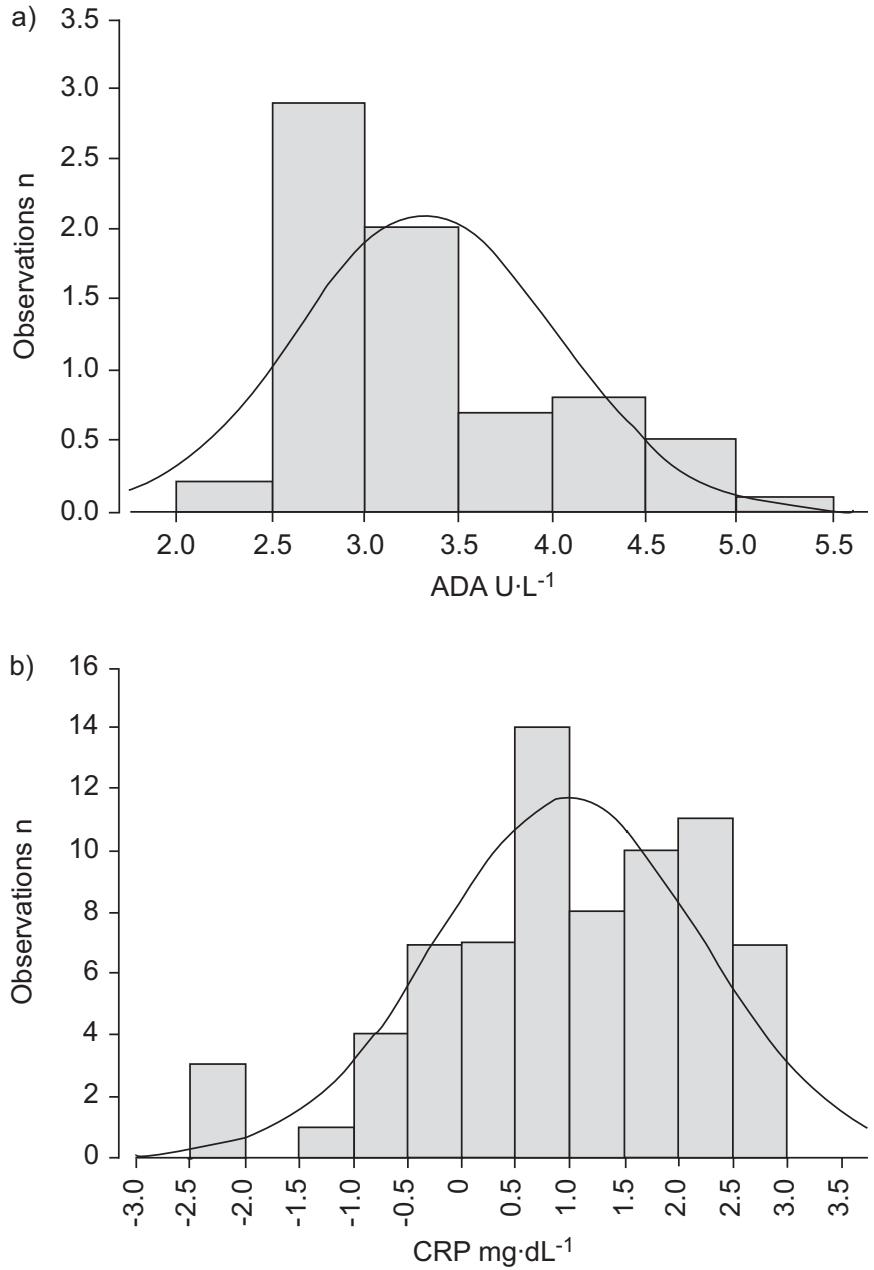

FIGURE 1. Distribution of a) adenosine deaminase (ADA) and b) C-reactive protein (CRP) after logarithmic transformation.

The respective estimated probability for classifying an individual as having MPE, TPE or PPE is as follows: $\mathrm{p}_{1}=\mathrm{e}^{9.23-0.16 \mathrm{ADA}-0.62 \mathrm{CRP}} / \mathrm{D}, \quad \mathrm{p}_{2}=\mathrm{e}^{0.47+0.06 \mathrm{ADA}-0.70 \mathrm{CRP}} / \mathrm{D}$ or $\mathrm{p}_{3}=1 / \mathrm{D}$, where $\mathrm{D}=1+\mathrm{e}^{9.23-0.16 \mathrm{ADA}-0.62 \mathrm{CRP}}+\mathrm{e}^{0.47+0.06 \mathrm{ADA}-0.70 \mathrm{CRP}}$. These probabilities predict the group membership of an individual. Combination of values below and above the cutoff points of the parameters CRP $\left(5.5 \mathrm{mg} \cdot \mathrm{dL}^{-1}\right)$ and ADA $\left(42.4 \mathrm{U} \cdot \mathrm{L}^{-1}\right)$ can be used to estimate the probability $\mathrm{p}_{\mathrm{i}}(\mathrm{i}=1-3)$, and thus to provide a crude rule for diagnosis. Table 8 shows the estimated values of $p_{i}(i=1-3)$ for various combinations of ADA and CRP. Thus an individual with an ADA concentration level of $>45 \mathrm{U} \cdot \mathrm{L}^{-1}$ and $\mathrm{CRP}$ concentration of $<4 \mathrm{mg} \cdot \mathrm{dL}^{-1}$ is more likely to belong to the TPE group, whereas one with an ADA concentration level of $<40 \mathrm{U} \cdot \mathrm{L}^{-1}$ and a CRP concentration of $>6 \mathrm{mg} \cdot \mathrm{dL}^{-1}$ is more likely to belong to the PPE group, and one with a CRP concentration of $<4 \mathrm{mg} \cdot \mathrm{dL}^{-1}$ to the MPE group.

The CVA discriminated the three groups successfully. Figure 3 shows the two-dimensional ordination form of CVA, which accounts for $71 \%$ of the total variation in the data. CV1 and CV2 were as follows: CV1 $=0.87 \mathrm{ADA}+0.16 \mathrm{CEA}+0.53 \mathrm{IL}-6+$ $0.41 \mathrm{IFN}-\gamma+0.52 \mathrm{VEGF}+0.26 \mathrm{CRP}+0.77 \mathrm{TNF}-\alpha$, and $\mathrm{CV} 2=0.17 \mathrm{ADA}$ $+0.02 \mathrm{CEA}+0.11 \mathrm{IL}-6+0.58 \mathrm{IFN}-\gamma+0.05 \mathrm{VEGF}+0.90 \mathrm{CRP}+0.15 \mathrm{TNF}-\alpha$,

\begin{tabular}{|c|c|c|c|c|c|c|c|}
\hline \multicolumn{2}{|c|}{ TABLE 3} & \multicolumn{6}{|c|}{$\begin{array}{l}\text { Spearman's correlation coefficients between the } \\
\text { seven parameters }\end{array}$} \\
\hline & ADA & CEA & IL-6 & IFN- $\gamma$ & VEGF & CRP & TNF-o \\
\hline ADA & 1.00 & & & & & & \\
\hline CEA & -0.33 & 1.00 & & & & & \\
\hline IL-6 & 0.51 & -0.39 & 1.00 & & & & \\
\hline IFN- $\gamma$ & 0.38 & -0.37 & 0.19 & 1.00 & & & \\
\hline VEGF & -0.18 & 0.23 & 0.16 & -0.28 & 1.00 & & \\
\hline CRP & 0.29 & -0.24 & 0.55 & 0.17 & 0.29 & 1.00 & \\
\hline TNF- $\alpha$ & 0.62 & -0.44 & 0.48 & 0.44 & -0.18 & 0.38 & 1.00 \\
\hline
\end{tabular}

respectively. The CV1 axis clearly separated MPE from TPE patients, whereas the CV2 axis separated MPE from PPE patients. In CV1 and CV2 equations, the largest coefficients were for $\mathrm{ADA}$ and $\mathrm{CRP}$, respectively, indicating that these parameters are the most important for discrimination relative to the others $[4,5]$.

\section{DISCUSSION}

The determination of biological marker levels in pleural effusions has been proposed as an alternative noninvasive means of establishing a diagnosis of pleural effusion. However, the use of these measurements in clinical practice remains controversial $[8,9]$. In the present prospective study, ADA, CRP, CEA, IFN- $\gamma$, IL- 6 , TNF- $\alpha$ and VEGF concentration levels were measured in pleural fluid obtained from patients with exudative pleural effusion. To the present authors' knowledge, this is the first study to attempt exploration of the use of the seven previously mentioned parameters, simultaneously, for discrimination between the three different causes of exudative pleural effusion (MPE, PPE and TPE).

\section{TABLE 4 Areas under the curve (AUCs) produced by receiver operating characteristic curve analysis}

TPE versus MPE and PPE

PPE versus MPE and TPE

$\begin{array}{lll}\text { ADA } & 0.94(0.89-1.00) & 0.70(0.55-0.84) \\ \text { CRP } & 0.57(0.44-0.70) & 0.92(0.84-1.00) \\ \text { CEA } & 0.17(0.06-0.27) & 0.34(0.22-0.46) \\ \text { IL-6 } & 0.81(0.70-0.91) & 0.82(0.72-0.92) \\ \text { IFN- } \gamma & 0.93(0.82-1.00) & 0.51(0.36-0.67) \\ \text { VEGF } & 0.14(0.05-0.24) & 0.69(0.55-0.83) \\ \text { TNF- } \alpha & 0.89(0.81-0.97) & 0.70(0.56-0.84)\end{array}$

Data are presented as AUC \% (95\% confidence interval). TPE: tuberculous pleural effusion; MPE: malignant pleural effusion; PPE: parapneumonic pleural effusion; ADA: adenosine deaminase; CRP: C-reactive protein; CEA: carcinoembryonic antigen; IL: interleukin; IFN: interferon; VEGF: vascular endothelial growth factor; TNF: tumour necrosis factor. 


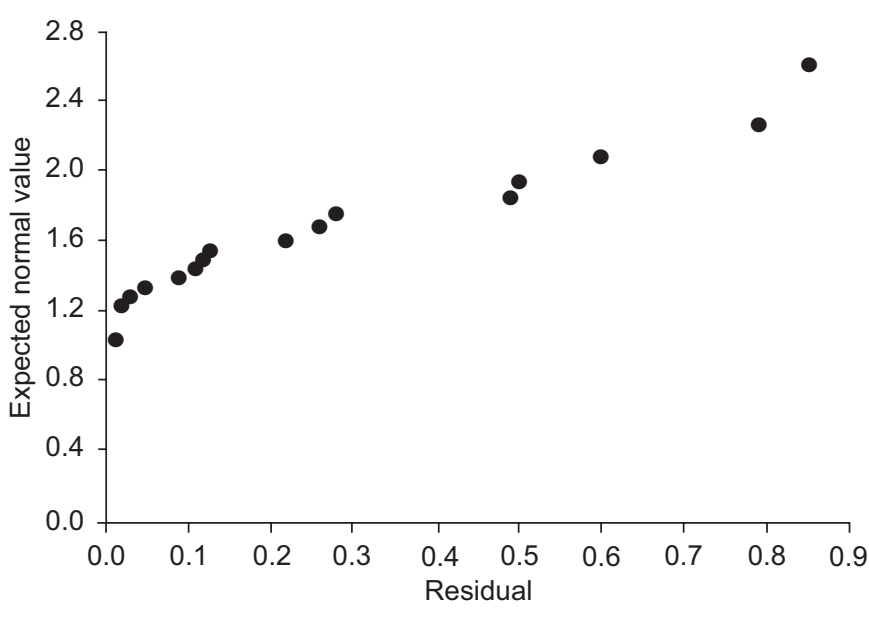

FIGURE 2. Half-normal plot of the residuals derived by fitting the multinomial logit model.

Pleural fluid ADA activity has been shown to be a valuable biochemical marker, which has a high sensitivity and specificity for the diagnosis of tuberculosis (TB) [9], but its diagnostic usefulness depends upon the local prevalence of TB, laboratory methodology, and population ethnicity. The only other parameter of comparable sensitivity and possibly higher specificity is IFN- $\gamma$ [11]. However, the latter's high cost and relatively long reaction time precludes its routine use [12]. A meta-analysis demonstrated that studies conducted in Europe showed significantly better diagnostic performance than those from other regions [13]. ADA combines low cost, easy performance and high diagnostic efficiency in the identification of TB pleurisy [14]. According to the present study, ADA provides the largest AUC (0.94) for the discrimination of TPE from MPE and PPE (cut-off point 42.4), and this finding is in agreement with previous studies [10]. In addition, when the logit model was fitted, ADA concentration level was found to be significant. IFN- $\gamma$ also provided a large AUC (0.93) for the discrimination of TPE from MPE and PPE. However, the logit model failed to show a significant role of this finding.

\begin{tabular}{lcccc}
\hline TABLE 5 & $\begin{array}{l}\text { Confusion matrix for multinomial logit model } \\
\text { predictions }\end{array}$ \\
$\begin{array}{l}\text { Observed } \\
\text { classification }\end{array}$ & \multicolumn{3}{c}{$\begin{array}{c}\text { Predicted } \\
\text { classification }\end{array}$} & $\begin{array}{c}\text { Correct classification } \\
\% \text { (95\% Cl) }\end{array}$ \\
\cline { 2 - 4 } & MPE & TPE & PPE & \\
\hline MPE & 43 & 0 & 2 & $95.6(90.8-100)$ \\
TPE & 0 & 11 & 1 & $91.7(85.3-98.1)$ \\
PPE & 4 & 1 & 10 & $66.7(55.8-77.6)$ \\
Overall \% & 65.3 & 16.7 & 18.1 & $88.9(81.6-96.2)$ \\
\hline
\end{tabular}

Data are presented as $n$, unless otherwise stated. MPE: malignant pleural effusion; TPE: tuberculous pleural effusion; PPE: parapneumonic pleural effusion; Cl: confidence interval.

\begin{tabular}{|c|c|c|c|c|c|}
\hline \multirow[t]{2}{*}{ TABLE 6} & \multicolumn{5}{|c|}{$\begin{array}{l}\text { Percentage of correctly classified individuals for } \\
\text { each parameter, and the effect of adding } \\
\text { markers to adenosine deaminase (ADA) and C- } \\
\text { reactive protein (CRP) in the logit model }{ }^{\#}\end{array}$} \\
\hline & & MPE & TPE & PPE & Overall \\
\hline \multicolumn{2}{|l|}{ ADA } & 97.8 & 75.0 & 20.0 & 77.8 \\
\hline \multicolumn{2}{|l|}{ CRP } & 95.6 & 0 & 73.3 & 75.0 \\
\hline \multicolumn{2}{|l|}{ IL-6 } & 91.1 & 0 & 53.3 & 68.1 \\
\hline \multicolumn{2}{|l|}{ IFN- $\gamma$} & 100 & 75.0 & 13.3 & 77.8 \\
\hline \multicolumn{2}{|l|}{ TNF- $\alpha$} & 93.3 & 0 & 46.7 & 68.1 \\
\hline \multicolumn{2}{|l|}{ CEA } & 88.9 & 41.7 & 0 & 62.5 \\
\hline \multicolumn{2}{|l|}{ VEGF } & 88.9 & 41.7 & 0 & 62.5 \\
\hline \multicolumn{2}{|c|}{ ADA/CRP } & 95.6 & 91.7 & 66.7 & 88.9 \\
\hline \multicolumn{2}{|c|}{ ADA/CRP/IL-6 } & 95.6 & 91.7 & 80.0 & 91.7 \\
\hline \multicolumn{2}{|c|}{ ADA/CRP/IFN- $\gamma$} & 97.8 & 91.7 & 80.0 & 93.1 \\
\hline \multicolumn{2}{|c|}{ ADA/CRP/TNF- $\alpha$} & 97.8 & 91.7 & 73.3 & 91.7 \\
\hline \multicolumn{2}{|c|}{ ADA/CRP/CEA } & 93.3 & 91.7 & 80.0 & 90.3 \\
\hline \multicolumn{2}{|c|}{ ADA/CRP/VEGF } & 95.6 & 91.7 & 80.0 & 91.7 \\
\hline \multicolumn{2}{|c|}{ ADA/CRP/IL-6/TNF- $\alpha$} & 97.8 & 91.7 & 80.0 & 93.1 \\
\hline \multicolumn{2}{|c|}{ ADA/CRP/IL-6/CEA } & 97.8 & 91.7 & 86.7 & 94.4 \\
\hline \multicolumn{2}{|c|}{ ADA/CRP/IL-6/VEGF } & 97.8 & 91.7 & 86.7 & 94.4 \\
\hline \multicolumn{2}{|c|}{ ADA/CRP/TNF- $\alpha / C E A$} & 93.3 & 91.7 & 80.0 & 90.3 \\
\hline \multicolumn{2}{|c|}{ ADA/CRP/TNF- $\alpha /$ VEGF } & 97.8 & 91.7 & 80.0 & 93.1 \\
\hline \multicolumn{2}{|c|}{ ADA/CRP/CEA/VEGF } & 97.8 & 91.7 & 93.3 & 95.8 \\
\hline
\end{tabular}

MPE: malignant pleural effusion; TPE: tuberculous pleural effusion; PPE: parapneumonic pleural effusion; IL: interleukin; IFN: interferon; TNF: tumour necrosis factor; CEA: carcinoembryonic antigen; VEGF: vascular endothelia growth factor. * : for the combinations ADA/CRP/IL-6/IFN- $\gamma$, ADA/CRP/IFN- $\gamma /$ TNF- $\alpha$, ADA/CRP/IFN- $\gamma / \mathrm{CEA}$ and ADA/CRP/IFN- $\gamma / \mathrm{NEGF}$, the model cannot be fitted; either the maximum likelihood estimates do not exist or some parameter estimates are infinite.

TNF- $\alpha$ is a pro-inflammatory cytokine that is known to regulate the growth and differentiation of a variety of immune cells. Increased levels of TNF- $\alpha$ have been found in both infectious pleural effusion $[9,15]$ and MPE [16]. PORCEL et al. [8] suggested that pleural TNF- $\alpha$ could be a biochemical marker of inflammation in patients with PPE. Additionally, elevated levels of pleural TNF- $\alpha$ identified the subgroup of patients with no purulent-appearing PPE who required invasive management with tube thoracostomy more reliably than traditional fluid chemistries [8]. However, others have reported no difference between TNF- $\alpha$ concentration level in exudative pleural effusion of various aetiologies [16]. In the present study, it was found that TNF- $\alpha$ concentration levels were more increased in TPE, but this finding was nonsignificant.

IL-6 has long been regarded as a pro-inflammatory cytokine induced by lipopolysaccharide along with TNF- $\alpha$ and IL-1. IL6 is often used as a marker of systemic activation of proinflammatory cytokines [17]. IL-6 has been found to be elevated in MPE, especially after pleurodesis [18]. However, conflicting results have been reported in distinguishing MPE from infectious pleural effusion by IL-6 concentration level [9]. According to the present findings, IL-6 concentration was increased in TPE and PPE, but these findings were nonsignificant after fitting the logit model. 


\begin{tabular}{|c|c|c|c|c|}
\hline \multirow[t]{2}{*}{ TABLE 7} & \multicolumn{4}{|c|}{$\begin{array}{l}\text { Detection rate (DR), false-positive rate (FPR) and } \\
\text { likelihood ratio \# (LR) for considering the effect of } \\
\text { adding markers to adenosine deaminase (ADA) } \\
\text { and C-reactive protein (CRP) in the logit model }\end{array}$} \\
\hline & & DR & FPR & LR \\
\hline \multicolumn{5}{|l|}{ ADA/CRP } \\
\hline MPE & & $43 / 45(96)$ & 4/27 (14.8) & 6.5 \\
\hline TPE & & 11/12 (92) & $1 / 60(1.7)$ & 54 \\
\hline PPE & & $10 / 15(67)$ & $3 / 57(5.3)$ & 12.6 \\
\hline \multicolumn{5}{|c|}{ ADA/CRP/IL-6 } \\
\hline MPE & & 43/45 (96) & 2/27 (7.4) & 12.9 \\
\hline TPE & & 11/12 (92) & $1 / 60(1.7)$ & 55 \\
\hline PPE & & $12 / 15(80)$ & 3/57 (5.3) & 15.2 \\
\hline \multicolumn{5}{|c|}{ ADA/CRP/IFN- $\gamma$} \\
\hline MPE & & $44 / 45(98)$ & $3 / 27(11.1)$ & 8.8 \\
\hline TPE & & 11/12 (92) & $0 / 60(0)$ & NA \\
\hline PPE & & $12 / 15(80)$ & 2/57 (3.5) & 22.8 \\
\hline \multicolumn{5}{|c|}{ ADA/CRP/TNF- $\alpha$} \\
\hline MPE & & $44 / 45(98)$ & $3 / 27(11.1)$ & 8.8 \\
\hline TPE & & 11/12 (92) & $1 / 60(1.7)$ & 55 \\
\hline PPE & & $11 / 15(73)$ & 2/57 (3.5) & 20.9 \\
\hline \multicolumn{5}{|c|}{ ADA/CRP/CEA } \\
\hline MPE & & $42 / 45(93)$ & $3 / 27(11.1)$ & 8.4 \\
\hline TPE & & 11/12 (92) & $1 / 60(1.7)$ & 55 \\
\hline PPE & & $12 / 15(80)$ & 3/57 (5.3) & 15.2 \\
\hline \multicolumn{5}{|c|}{ ADA/CRP/VEGF } \\
\hline MPE & & $43 / 45(96)$ & $3 / 27(11)$ & 8.6 \\
\hline TPE & & 11/12 (92) & $1 / 60(2)$ & 55 \\
\hline PPE & & $12 / 15(80)$ & 2/57 (4) & 22.8 \\
\hline \multicolumn{5}{|c|}{ ADA/CRP/IL-6/TNF- $\alpha$} \\
\hline MPE & & $44 / 45(98)$ & 2/27 (7.4) & 13.2 \\
\hline TPE & & 11/12 (92) & 1/60 (1.7) & 55 \\
\hline PPE & & $12 / 15(80)$ & 2/57 (3.5) & 22.8 \\
\hline \multicolumn{5}{|c|}{ ADA/CRP/IL-6/CEA } \\
\hline MPE & & $44 / 45$ (98) & 2/27 (7.4) & 13.2 \\
\hline TPE & & 11/12 (92) & 0/60 (0) & NA \\
\hline PPE & & $13 / 15(87)$ & 2/57 (3.5) & 24.7 \\
\hline \multicolumn{5}{|c|}{ ADA/CRP/IL-6/VEGF } \\
\hline MPE & & $44 / 45(98)$ & 2/27 (7) & 13.2 \\
\hline TPE & & 11/12 (92) & 0/60 (0) & NA \\
\hline PPE & & $13 / 15(87)$ & 2/57 (4) & 24.7 \\
\hline \multicolumn{5}{|c|}{ ADA/CRP/TNF- $\alpha /$ CEA } \\
\hline MPE & & $42 / 45(93)$ & $3 / 27(11.1)$ & 8.4 \\
\hline TPE & & 11/12 (92) & $1 / 60(1.7)$ & 55 \\
\hline PPE & & $12 / 15(80)$ & $3 / 57(5.3)$ & 15.2 \\
\hline \multicolumn{5}{|c|}{ ADA/CRP/TNF- $\alpha /$ VEGF } \\
\hline MPE & & $44 / 45$ (98) & $3 / 27(11)$ & 8.8 \\
\hline TPE & & 11/12 (92) & $0 / 60(0)$ & NA \\
\hline PPE & & 12/15 (80) & 2/57 (4) & 22.8 \\
\hline \multicolumn{5}{|c|}{ ADA/CRP/CEA/VEGF } \\
\hline MPE & & 44/45 (98) & $1 / 27(4)$ & 26.4 \\
\hline TPE & & 11/12 (92) & 0/60 (0) & NA \\
\hline PPE & & 14/15 (93) & 2/57 (4) & 26.6 \\
\hline
\end{tabular}

Data are presented as $n(\%)$, unless otherwise stated. MPE: malignant pleural effusion; TPE: tuberculous pleural effusion; PPE: parapneumonic pleural effusion; IL: interleukin; IFN: interferon; TNF: tumour necrosis factor; CEA: carcinoembryonic antigen; VEGF: vascular endothelial growth factor; NA: not applicable. * : DR/FPR.

\begin{tabular}{|c|c|c|c|c|}
\hline \multirow{2}{*}{$\begin{array}{l}\text { TABLE } 8 \\
\text { ADA U.L } L^{-1}\end{array}$} & \multicolumn{4}{|c|}{$\begin{array}{l}\text { Estimated probabilities for various combinations } \\
\text { of adenosine deaminase (ADA) and C-reactive } \\
\text { protein (CRP) }\end{array}$} \\
\hline & CRP $\mathrm{mg} \cdot \mathrm{dL}^{-1}$ & p1 & p2 & p3 \\
\hline 45 & 4.0 & 0.19 & 0.51 & 0.31 \\
\hline $149.7^{\#}$ & $0.1^{\circ}$ & 0.00 & 1.00 & 0.00 \\
\hline 40 & 6.0 & 0.23 & 0.17 & 0.59 \\
\hline $10.6^{\circ}$ & $17.1^{\#}$ & 0.04 & 0.00 & 0.96 \\
\hline 40 & 4.0 & 0.38 & 0.34 & 0.28 \\
\hline $10.6^{*}$ & $0.1^{\circ}$ & 1.00 & 0.00 & 0.00 \\
\hline \multicolumn{5}{|c|}{$\begin{array}{l}\text { p1: probability that an individual belongs to the malignant pleural effusion } \\
\text { group; p2: probability that an individual belongs to the tuberculous pleural } \\
\text { effusion group; p3: probability that an individual belongs to the parapneumonic } \\
\text { pleural effusion group. }{ }^{\#} \text { : maximum observed value; " minimum observed } \\
\text { value. }\end{array}$} \\
\hline
\end{tabular}

CRP is an acute-phase protein widely used as a marker of inflammation and tissue injury [19]. CRP concentration level has been studied in pleural fluid and been found to be higher in benign than in malignant exudates [20]. TURAY et al. [21] have shown that pleural fluid CRP concentration levels of $>30 \mathrm{mg} \cdot \mathrm{L}^{-1}$ show a high sensitivity $(93.7 \%)$ and specificity $(76.5 \%)$ and a positive predictive value of $98.4 \%$ for the diagnosis of PPE [21]. The present results show that CRP provides the largest AUC (0.92) for discrimination of PPE from MPE and TPE, and it was a significant parameter in discriminating between the groups.

Several studies have confirmed the presence of high concentration levels of VEGF in exudative, especially malignant and inflammatory, effusions [22, 23]. Finally, the conclusion arising from the literature is that there is an overlap between VEGF concentration levels in the various groups of pleural effusion; therefore, VEGF concentration levels are unlikely to be diagnostically useful [22]. The present study has demonstrated that VEGF concentration levels are increased in both MPE and PPE, and that VEGF is not a significant parameter for discrimination between the three groups.

Analysis of tumour marker levels in serum has been applied to the diagnosis, prognosis and treatment of patients with lung cancer. Furthermore, pleural fluid from patients with MPE is known to contain detectable levels of tumour markers. CEA was one of the first markers to be evaluated in lung cancer [24]. Determination of CEA concentration can be used as a diagnostic tool for MPE since $40-70 \%$ of pleural fluids give positive CEA assay results [25]. In accordance with previous reports, CEA was found to be the best single tumour marker in pleural fluid [26, 27]. However, although CEA has been studied the most and been shown to be very specific, its sensitivity remains in the region of $29-77 \%$, with variable cutoff values. LEE et al. [28] demonstrated that, in cases of suspicious MPE showing a negative cytology, particularly in the absence of a visible tumour and/or unsuitability for invasive procedures, the determination of tumour marker levels in the pleural fluid might be helpful as a complementary tool for the differential diagnosis of pleural effusion. Finally, 


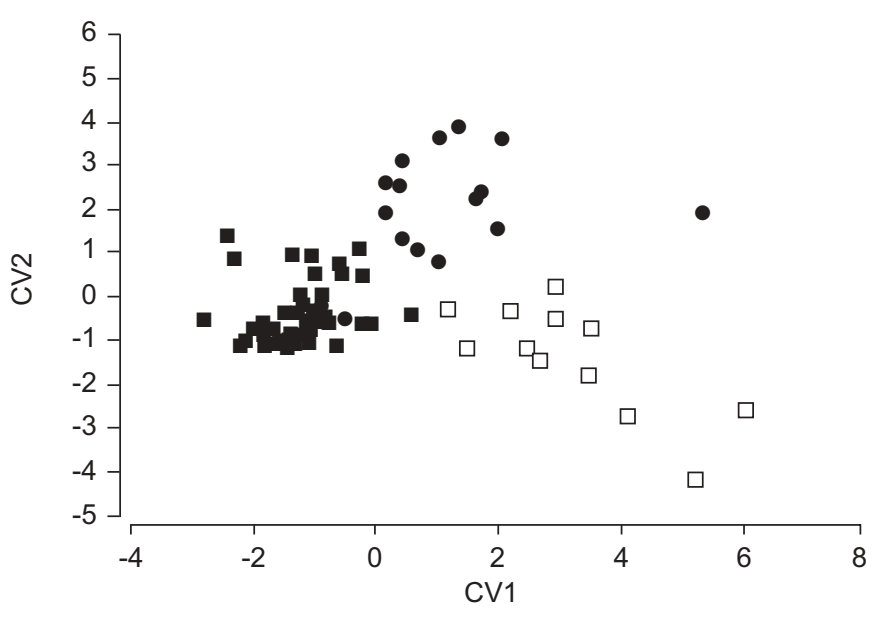

FIGURE 3. Two-dimensional ordination form of canonical variate (CV) analysis. - parapneumonic pleural effusion; $\square$ : tuberculous pleural effusion; malignant pleural effusion.

although an elevated concentration level of CEA in pleural fluid is suggestive of malignancy, CEA can be elevated in $9 \%$ of pleurisy owing to benign diseases, especially empyemas and complicated PPEs. Identification of the most frequent causes of false-positive results using CEA helps to correctly interpret the findings of this tumour marker [29]. In the present study, CEA concentration levels were highest in MPEs. However, a positive significance of this parameter was not found in the simultaneous system.

In the present study, the number of cases might be considered relatively low compared with the number of parameters used for discrimination. Although, in general, small sample size tends to result in inefficient discrimination, the discrimination accuracy does not strictly depend upon sample size but rather upon the nature (distribution) of the data, variability of the data and number of variables examined [30-32]. Nevertheless, in order to strengthen further the utility of the multinomial logit model and the derived classification rule, CVA was applied to the data. CVA verified the existence of three distinct groups and the discriminative value of mainly ADA and CRP, although the importance of most of the CV1 parameters should also be considered. However, accumulation of more data would enable further exploration of the validity of the discrimination results by application of additional techniques, such as classification trees [33]. When a small dataset is used to both estimate the model parameters and test the model, it is to be expected that the results are favourable. Ideally, two independent datasets should be used, one to derive the parameters and another to test them. Alternatively, there are more complex methods of parameter estimation, such as bootstrapping [34], but estimation by this method is beyond the scope of the present article.

In conclusion, combinations of markers can be useful in the discrimination of exudative pleural effusion groups. Although, the present analysis focused on adenosine deaminase and Creactive protein, the data presented in the current study do not exclude the usefulness of one or more additional markers. Therefore, further and larger studies should not focus only on adenosine deaminase and C-reactive protein, and the results should be synthesised to provide more evidence.

\section{REFERENCES}

1 Marel M, Zrustova M, Stasny B, Light RW. The incidence of pleural effusion in a well-defined region. Epidemiologic study in central Bohemia. Chest 1993; 104: 1486-1489.

2 Giusti G. Adenosine deaminase. In: Methods of Enzymatic Analysis. Bergmeyer HU, ed. New York, Academic Press, 1974; pp. 1092-1099.

3 Zintzaras E, Germenis AE. Diagnostic performance of antibodies against tissue transglutaminase for the diagnosis of coeliac disease: meta-analysis. Clin Vaccine Immun 2006; 13: 15-28.

4 Jobson JD. Applied Multivariate Data Analysis. New York, Springer, 1992.

5 Zintzaras E, Brown NP, Kowald A. Growing a classification tree using the apparent misclassification rate. Comput Appl Biosci 1994; 10: 263-271.

6 Zintzaras E, Margaritopoulos J, Tsitsipis J. Statistical tree classification of aphids based on morphological characters. Comp Electron Agric 1999; 24: 165-175.

7 Zintzaras E. Statistical assessment of the synergistic effect between a vaccine and a drug. J Pharm Pharmacol 1992; 44: 873-874.

8 Porcel JM, Vives M Esquerda A. Tumor necrosis factor- $\alpha$ in pleural fluid. A marker of complicated parapneumonic effusions. Chest 2004; 125: 160-164.

9 Yokoyama A, Kohno N, Fujino S, Abe M, Ishida O, Hiwada K. Soluble interleukin-6 receptor levels in pleural effusions. Respir Med 1996; 90: 329-332.

10 Perez-Rodriguez E, Perez Walton IJ, Sanchez Hernandez JJ, et al. $\mathrm{ADA}_{1} / \mathrm{ADAp}$ ratio in pleural tuberculosis: an excellent diagnostic parameter in pleural fluid. Respir Med 1999; 93: 816-821.

11 Villegas MV, Labrada LA, Saravia MG. Evaluation of polymerase chain reaction, adenosine deaminase, and interferon $\gamma$ in pleural fluid for differential diagnosis of pleural tuberculosis. Chest 2000; 118: 1355-1364.

12 Ferrer J. Pleural tuberculosis. Eur Respir J 1997; 10: 942-947.

13 Goto M, Noguchi Y, Koyama H, Hira K, Shimbo T, Fukui T. Diagnostic value of adenosine deaminase in tuberculous pleural effusion: a meta-analysis. Ann Clin Biochem 2003; 40: 374-381.

14 Chen ML, Yu WC, Lam CW, Au KM, Kong FY, Chan A. Diagnostic value of pleural fluid adenosine deaminase activity in tuberculous pleurisy. Clin Chim Acta 2004; 341: 101-107.

15 Barnes PF, Fong SJ, Brennan PJ, Twomey PE, Mazumder A, Modlin RL. Local production of tumor necrosis factor and IFN- $\alpha$ in tuberculous pleuritis. J Immunol 1990; 145: 149-154.

16 Soderblom T, Nyberg P, Teppo AM, Klockars M, Riska H, Petterson T. Pleural fluid interferon- $\gamma$ and tumour necrosis factor- $\alpha$ in tuberculous and rheumatoid pleurisy. Eur Respir J 1996; 9: 1652-1655.

17 Opal SM, DePalo VA. Anti-inflammatory cytokines. Chest 2000; 117: 1162-1172.

18 Agrenius V, Gustafsson LE, Widstrom O. Tumour necrosis factor- $\alpha$ and nitric oxide, determined as nitrite, in malignant pleural effusion. Respir Med 1994; 88: 743-748. 
19 Okamura JM, Miyagi JM, Terada K, Hokama Y. Potential clinical applications of C-reactive protein. J Clin Lab Anal 1990; 4: 231-235.

20 Castano Vidriales JL, Amores Antequera C. Use of pleural fluid C-reactive protein in laboratory diagnosis of pleural effusions. Eur J Med 1992; 1: 201-207.

21 Turay UY, Yildirim Z, Turkoz Y, et al. Use of pleural fluid C-reactive protein in diagnosis of pleural effusions. Respir Med 2000; 94: 432-435.

22 Chang D-S, Rodriguez RM, Perkett EA, et al. Vascular endothelial growth factor in pleural fluid. Chest 1999; 116: 760-765.

23 Thickett DR, Armstrong L, Millar AB. Vascular endothelial growth factor (VEGF) in inflammatory and malignant pleural effusions. Thorax 1999; 54: 707-710.

24 Niklinski J, Furman M. Clinical tumour markers in lung cancer. Eur J Cancer Prev 1995; 4: 129-138.

25 Sculier JP, Body JJ, Jacobowitz D, Fruhling J. Value of CEA determination in biological fluids and tissues. Eur J Cancer Clin Oncol 1987; 23: 1091-1093.

26 Ferrer J, Villarino MA, Encabo G, et al. Diagnostic utility of CYFRA 21-1, carcinoembryonic antigen, CA 125, neuron specific enolase, and squamous cell antigen level determinations in the serum and pleural fluid of patients with pleural effusions. Cancer 1999; 86: 1488-1495.

27 Villena V, Lopez-Encuentra A, Echave-Sustaeta J, Martin-Escribano P, Ortuno-de-Solo B, Estenoz-Alfano
J. Diagnostic value of CA 72-4, carcinoembryonic antigen, CA 15-3, and CA 19-9 assay in pleural fluid: a study of 207 patients. Cancer 1996; 78: 736-740.

28 Lee JH, Chang JH. Diagnostic utility of serum and pleural fluid carcinoembryonic antigen, neuron-specific enolase, and cytokeratin 19 fragments in patients with effusions from primary lung cancer. Chest 2005; 128: 2298-2303.

29 Garcia-Pachon E, Padilla-Navas I, Dosda D, MirallesLlopis A. Elevated level of carcinoembryonic antigen in nonmalignant pleural effusions. Chest 1997; 111: 643-647.

30 Raudys S, Pikelis V. On dimensionality, sample size, classification error and complexity of classification algorithm in pattern recognition. IEEE Trans Pattern Anal Mach Intell 1980; 2: 242-252.

31 Wharton SW. An analysis of the effects of sample size on classification performance of a histogram based cluster analysis procedure. Pattern Recognit 1984; 17: 239-244.

32 Boulesteix AL, Tutz G, Strimmer K. A CART-based approach to discover emerging patterns in microarray data. Bioinformatics 2003; 18: 2465-2472.

33 Zintzaras E, Brown NP, Kowald A. Non-parametric classification of secondary protein structures. Comput Biol Med 2005; 36: 145-156.

34 Zintzaras E, Bouka P, Kowald A. Biometrical evaluation of bioequivalence trials using a bootstrap individual direct curve comparison method. Eur J Drug Metab Pharmacokinet 2002; 27: 11-16. 